\title{
Current studies of biomarkers for the early diagnosis of rheumatoid arthritis
}

This article was published in the following Dove Press journal:

Current Biomarker Findings

I September 2014

Number of times this article has been viewed

\section{S Chandrashekara}

ChanRe Rheumatology and Immunology Center and Research, Basaveshwaranagar, Bangalore, India
Correspondence: S Chandrashekara ChanRe Rheumatology and Immunology Center and Research, \#149, 15th Main, NHCL Water Tank Road, 4th Block, 3rd stage, Basaveshwaranagar, Bangalore-560079, India Tel +9l 080425 I 6699 Fax +9| 080 425I 6600 Email chandrashekara_s@yahoo.com
Abstract: Early treatment of rheumatoid arthritis (RA) with disease-modifying antirheumatic drugs will improve the outcome significantly. Early diagnosis of RA continues to be a challenge. The disease needs to be distinguished from other self-limiting arthritis and connective tissue disease. Currently available autoantibodies like rheumatoid factors and anticitrullinated cyclic peptide have limited sensitivity and specificity. RA, being a heterogeneous disease, with no unique or distinct defect that has been described, is less likely to have a single pathognomonic marker. There are defined predisposing genetic factors, cell characteristics, cytokine changes, autoantibodies, and products of disease process that have been demonstrated to distinguish rheumatoid from normal and other arthritis. Studies have demonstrated that combinations of factors allow for more specific RA diagnosis; however, when considerations are given to the factors separately, sensitivity increases at the cost of specificity. The present review briefly describes the value of some of the candidate factors and their combinations as diagnostic markers of early RA. Well-designed multicenter studies to evaluate these combinations using a scoring system are recommended for the development of precise and widely applicable biomarkers for early diagnosis of RA.

Keywords: autoantibodies, combination, early RA, specificity

\section{Introduction}

Rheumatoid arthritis (RA) affects $1 \%$ of the population across the globe. The disease, being a chronic erosive synovitis with slow progression into systemic disease, results in significant impairment in quality of life and reduces life expectancy. The current evidence in the treatment of RA has demonstrated that early and effective intervention will reduce the incidence of deformities, and, in a few instances, a long lasting remission. ${ }^{1}$ RA has to be differentiated, during early clinical presentation, from self-limiting viral arthritis, reactive arthritis, and even persisting nonerosive synovitis of systemic lupus erythematosus. Often, the self-limiting true RA needs to be identified, since it may not need long-term disease modifying antirheumatic drugs. Early arthritis clinics across the world have observed the following facts: 1 ) more than $50 \%$ of the patients who have synovitis at presentation resolve spontaneously over a period of time; ${ }^{2,3}$ and 2) the remaining patients with chronic progressive arthritis may develop a well-defined syndrome, such as RA or connective tissue disease, or continue to experience undifferentiated arthritis. ${ }^{2,4,5}$ The current challenge is to differentiate these two types of patients, in order to treat them appropriately. Inflammation is present in all the forms of arthritis and, hence, the markers of inflammation become nonspecific to differentiate inflammatory arthritis. However, the characteristic features which differentiate these two types, in earlier presentation of arthritis, will greatly assist in improving the 
care of RA with early interventions. This factor makes early diagnostic biomarkers one of the actively researched areas in management of RA.

A diagnostic biomarker, by definition, should be an objective, quantifiable characteristic of biological processes which helps in accurate diagnosis of the condition. It should consist of the following three characteristics: 1) analytical validation; 2) qualification; and 3) utilization. The current review attempts to analyze the available biomarkers for diagnosis of early RA.

\section{Biomarkers in early RA: complexities}

It is worthwhile to explore the complexity of the disease, before taking up an analysis of the biomarkers for early diagnosis of RA. RA is a heterogeneous disease in its clinical presentation as well as in its serological markers. The disease may vary from a very mild, self-limiting disease to an aggressive one with many different systemic involvements. Persisting inflammation, predominantly in the joints, is the hallmark of RA. However, simultaneous inflammation in areas other than joints cannot be excluded during the pathological process of RA. The defects in RA occur at three levels culminating in persisting inflammation. ${ }^{6}$ The defect in RA can be at three different levels: level 1 - the site of inflammation, such as synovium, where the autoreactive and inflammatory cells proliferate; level 2 - the interface of regulatory processes, such as peripheral tolerance mechanism; and level 3 - the central level of regulation of autoimmunity. These levels are arbitrary and often overlap; all possible defects of these levels are described in RA. Another major challenge is that the changes triggering the disease and the subsequent process of development may differ between patient groups. Presumably, the changes at the regulatory levels leading to persistent inflammation, and those inhibiting reversible processes, may be unique to RA. However, changes in RA described in published literature, and the published biological modeling based on them, appear to be cumulative, and the clinical characteristics of presentation of RA may be proportionate to the pathological process (defects). There is no unique defect described in RA to date, which could account for the development and perpetuation of the disease; additionally, RA develops over a period of time. As a simple example, studies on rheumatoid factor (RF) as well as antibody to the cyclic citrullinated peptide (CCP) (anti-CCP) have demonstrated appearance of RF and anti-CCP in circulation well before disease development in a significant percentage of patients. ${ }^{7}$ It is also observed that many patients with the antibody may not develop the disease, and a significant proportion of diagnosed RA patients do not develop these antibodies to a detectable limit. Hence, it is unlikely that a single specific marker represents the complex stepwise errors occurring in the development of RA. The probable steps leading to the development and propagation of RA are represented in Figure 1. Current understanding of the therapy and a need for early intervention for better outcome of RA dictate the search for an ideal biomarker that can accurately predict and help to subclassify the disease activity, in order to plan an effective intervention.

Factors constituting the various steps, along with the probable constituents of the steps involved, as described in the literature, and the proposed mechanism of development of disease are shown in Figure 2. A few of the parameters described in published literature are depicted in the figure at their probable location in the disease process. A major limitation of this representation is that the flow and sequence of pathological process is not as simple and straightforward as represented in the figure. As appraised by various

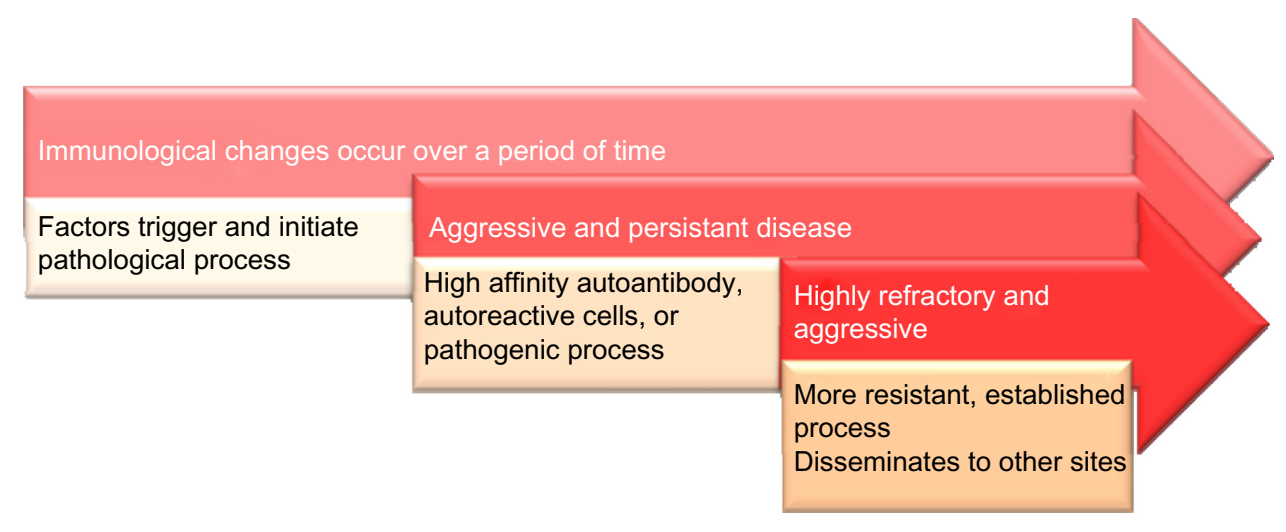

Figure I Development and propagation of RA pathological process. Abbreviation: RA, rheumatoid arthritis. 


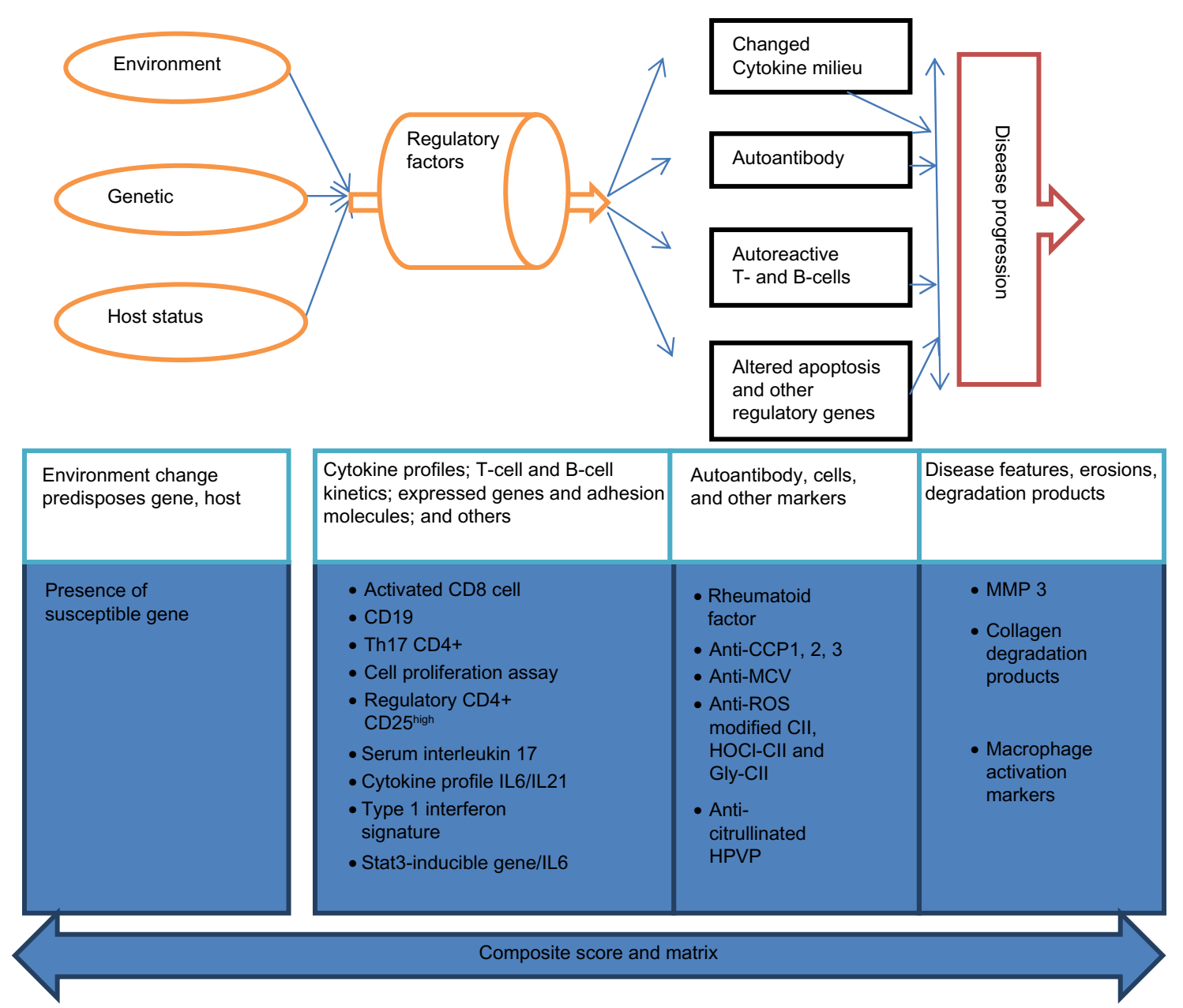

Figure 2 Proposed scheme of biological events in the development of early arthritis, showing probable location of potential biomarkers in the pathological process. Abbreviations: CCP, cyclic citrullinated peptide; CII, type II collagen; Gly, glycation/glycoxidation; HOCI, hypochlorous acid; HPVP, human papilloma virus peptide; IL, interleukin; MCV, mutated citrullinated vimentin; MMP, matrix metalloprotinase; ROS, reactive oxygen species; Th, $T$ helper.

publications, autoantibodies may be present in very early courses of the disease. ${ }^{7}$

In autoimmune diseases including RA, inflammation is the ultimate pathological process which causes damages and explains the majority of clinical manifestations. Hence, measuring inflammation and the markers representing inflammation can serve as a marker of disease activity, rather than representing the unique nature of the disease. Thus, a diagnostic biomarker of inflammation will not serve as a specific marker, as RA is a multifaceted disease represented by its clinical characteristics and pathological processes. Table 1 represents the factors that form the basis of this heterogeneity. The processes include dysregulation of proinflammatory factors, anti-inflammatory factors, or both. The heterogeneity of the disease has been attributed to differences in the activation of immune competent cells, cytokines, and expression of different receptors, partially or completely influenced by genetic status. ${ }^{8-12}$ In view of these perspectives, identifying a single biomarker to diagnose RA is theoretically not possible.

Studies exploring single biomarkers such as an autoantibody have limited sensitivity, but a few of them have a good specificity. In contrast, studies of genetic polymorphism and clinical severity have demonstrated that a combination of biomarkers have more predictive strength than single biomarkers. IL-6 and IL-10 genotype polymorphism along with presence of RF and antiCCP, ${ }^{13}$ TNF polymorphism with development of disease in familial clusters, and HLA DRB1 shared epitope and anti-CCP ${ }^{14}$ in development of disease are good examples of such combination of biomarkers. Consequently, a single biomarker may not sufficiently predict or identify all of the early RA patients. We present here a review of various preliminary studies dealing with the specificity and sensitivity of several possible markers, and the current status of these biomarkers. 
Table I Factors responsible for the heterogeneity of rheumatoid arthritis

\begin{tabular}{|c|c|}
\hline SI number & Disease feature \\
\hline I & $\begin{array}{l}\text { Chronology } \\
\text { Acute } \\
\text { Subacute } \\
\text { Chronic }\end{array}$ \\
\hline 2 & $\begin{array}{l}\text { Synovium pathology } \\
\text { Diffuse synovitis } \\
\text { Follicular Synovitis } \\
\text { Nonerosive synovitis } \\
\text { Granulomatous synovitis }\end{array}$ \\
\hline 3 & $\begin{array}{l}\text { Erosive disease } \\
\text { Nonerosive } \\
\text { Erosive }\end{array}$ \\
\hline 4 & $\begin{array}{l}\text { Antibody positivity } \\
\text { Seronegative } \\
\text { RF positive } \\
\text { Anti-CCP positive }\end{array}$ \\
\hline 5 & $\begin{array}{l}\text { Extra-articular features } \\
\text { Sicca symptoms } \\
\text { Nodules } \\
\text { Vasculitis } \\
\text { Felty's syndrome } \\
\text { Interstitial lung disease }\end{array}$ \\
\hline 6 & $\begin{array}{l}\text { Responsiveness to drugs } \\
\text { Refractory to DMARD } \\
\text { Responsive to DMARD }\end{array}$ \\
\hline 7 & Cell and cytokine profile \\
\hline
\end{tabular}

Abbreviations: $C C P$, cyclic citrullinated peptide; DMARD, disease-modifying antirheumatic drug; RF, rheumatoid factor; Sl, serial.

\section{Biomarkers in early RA: current scenario}

As evident from Figure 2, cell numbers, characteristics and functions, different autoantibodies, cytokine levels, disease features by imaging, and products of disease process that appear at different stages of the progression of RA have been described and their utility as diagnostic markers of RA has been explored. The rest of the article provides a summary of the current state of each class of potential markers. Tables 2-5 present the specificity and sensitivity of some of the biomarkers from current published data, and the biomarkers are grouped according to their position in the scheme (Figure 2).

\section{Environmental and genetic markers for the risk of RA}

The presence of a risk factor in patients presenting with arthritis can serve as a predictive marker or as a factor to help in confirming the diagnosis. The studies which have looked into the gene expression signature in CD4 cells could reasonably classify the patients who develop RA subsequently. ${ }^{15}$ However, the test performed better in the anti-citrullinated peptide antibody (ACPA)-negative subset than the positive subset. The changes in CD4 cells have been described to be specific to the ACPA-negative group. In analysis of the susceptible epitope (SE) genes, which were defined as $D R$ $B 1 * 0404$ and $D R B 1 * 0401$ when analyzed in both the patients and controls, the respective sensitivity and specificity figures for carriers of two SE genes were 28\% (16/57) and 95\%, respectively. The specificity for the $B 1 * 0401$ allele (74\%) was higher than that for SE given either $B 1 * 0401$ or $B 1 * 0404$, but the sensitivity increased to $60 \%$. The frequencies of the presence of one or both of the SE genes studied in pre-RA patients were significantly greater than in the controls. ${ }^{16}$ The PTPN22 1858C/T polymorphism was originally associated with type 1 diabetes, and later with other autoimmune diseases and RA. The PTPN-22 CT + TT was positive in 39.3\% of 35 pre-patients of RA when prescreened, compared to only $19.7 \%$ of 71 normal controls. This estimated a sensitivity of

Table 2 Genetic factors as potential markers of RA with available evidence

\begin{tabular}{|c|c|c|c|c|}
\hline $\begin{array}{l}\text { Markers of early RA } \\
\text { (reference) }\end{array}$ & $\begin{array}{l}\text { Specificity } \\
\text { (\%) }\end{array}$ & $\begin{array}{l}\text { Sensitivity } \\
\text { (\%) }\end{array}$ & $\begin{array}{l}\text { Positivity } \\
(\%)\end{array}$ & $\begin{array}{l}\text { Observation/ } \\
\text { detection }\end{array}$ \\
\hline $\begin{array}{l}\text { I 2-gene transcriptional signature } \\
\left.\text { (Pratt et } \mathrm{al}^{15}\right)\end{array}$ & & & & - \\
\hline Overall $(n=\mid I I)$ & 70 & 68 & na & \\
\hline ACPA subset $(n=49)$ & 75 & 85 & 26.5 & \\
\hline $\begin{array}{l}\text { SE HLA DR allele } \\
\left(\text { Berglin et } \mathrm{a}^{16}\right)\end{array}$ & & & & - \\
\hline $\begin{array}{l}\text { SE HLA BI*0404/040I } \\
\text { (Johansson et } \mathrm{al}^{17} \text { ) }\end{array}$ & 64 & 60 & na & \\
\hline $\mathrm{SE}(B \mid * 0404$ or $040 I)$ & 61.8 & 55.6 & na & \\
\hline $\begin{array}{l}\text { SNP PTPN22 gene } \\
\text { (Johansson }^{17} \text { ) }\end{array}$ & & & & - \\
\hline PTPN22 CT + TT & 80.3 & 39.3 & na & \\
\hline
\end{tabular}

Abbreviations: ACPA, anti-cyclic citrullinated peptide antibody; HLA, human leukocyte antigen; na, not available; PTPN22, protein tyrosine phosphatase nucleotide 22; RA, rheumatoid arthritis; SE, susceptible epitope; SNP, single nucleotide polymorphism. 
Table 3 Regulatory factors as potential markers of RA with available evidence

\begin{tabular}{|c|c|c|c|c|}
\hline $\begin{array}{l}\text { Markers of early RA } \\
\text { (reference) }\end{array}$ & $\begin{array}{l}\text { Specificity } \\
\text { (\%) }\end{array}$ & $\begin{array}{l}\text { Sensitivity } \\
\text { (\%) }\end{array}$ & $\begin{array}{l}\text { Positivity } \\
\text { (\%) }\end{array}$ & Observation/detection \\
\hline $\begin{array}{l}\text { Activated CD8 cells } \\
\left.\text { (van Baarsen et al }{ }^{19}\right) \\
\text { Early RA* }\end{array}$ & na & na & 40 & $\begin{array}{l}\text { Median } 33.1 \%(23.6-42.9 \%) \text { for early RA versus } \\
22.2 \% \text { (I5.75-26.55\%) for healthy controls }\end{array}$ \\
\hline $\begin{array}{l}\text { CDI9 B-cells } \\
\left(\text { van Baarsen et al }{ }^{19}\right) \\
\text { Early RA* } \\
\text { At risk* }\end{array}$ & $\begin{array}{l}\text { na } \\
\text { na }\end{array}$ & $\begin{array}{l}\text { na } \\
\text { na }\end{array}$ & $\begin{array}{l}37 \\
40\end{array}$ & $\begin{array}{l}\text { Median } 38.45 \%(21.45-48.90 \%) \text { for early RA versus } \\
20.05 \% \text { (13.03-28.25\%) for healthy controls }\end{array}$ \\
\hline $\begin{array}{l}\text { ThI7 CD4+ T-cells } \\
\text { (Arroyo-Villa et } \mathrm{al}^{21} \text { ) }\end{array}$ & na & na & na & $\begin{array}{l}\text { Significantly lower \% of circulating Th I } 7 \text { cells and lower } \\
\text { CD4-derived IL- I } 7 \text { secretion were observed, relative to } \\
\text { healthy controls, but only for anti-CCP-positive patients }\end{array}$ \\
\hline $\begin{array}{l}\text { T-cell proliferation kinetics } \\
\left(\text { Pawłowska et } \mathrm{a}^{22} \text { ) }\right. \\
\text { Cell-cycle duration } \\
\mathrm{G}_{0}-\mathrm{G}_{\text {, transition time }} \\
\text { Ratio of cell divisions } \\
\text { Ratio of dividing cells }\end{array}$ & $\begin{array}{l}92.5 \\
89 \\
97.5 \\
74.1\end{array}$ & $\begin{array}{l}80 \\
95.5 \\
60 \\
95.5\end{array}$ & $\begin{array}{l}\text { na } \\
\text { na } \\
\text { na } \\
\text { na }\end{array}$ & $\begin{array}{l}\text { Proliferation factors show higher specificity and } \\
\text { sensitivity in the detection of early RA compared to } \\
\text { routine serological tests }\end{array}$ \\
\hline $\begin{array}{l}\text { Reg CD4+ CD25 } 5^{\text {high }} \text { T-cell } \\
\left(\text { Lawson et } \mathrm{a}^{20}\right)\end{array}$ & $62.6^{*}$ & $37.4^{*}$ & na & $\begin{array}{l}\text { Smaller proportion of CD4+CD25 high } \mathrm{T} \text {-cells in the } \\
\text { peripheral blood of early active RA patients (mean } 4.25 \% \text { ) } \\
\text { than in healthy controls (mean } 5.3 \%, P=0.00 \mathrm{I} \text { ) }\end{array}$ \\
\hline $\begin{array}{l}\text { Serum IL-7 } \\
\left(\text { Goëb et } \mathrm{al}^{23}\right)\end{array}$ & & & & $\begin{array}{l}\text { Serum levels of IL-7 are reduced in both early and } \\
\text { established disease }\end{array}$ \\
\hline $\begin{array}{l}\text { Overall } \\
\text { ACPA }\end{array}$ & $\begin{array}{l}84.5 \\
83\end{array}$ & $\begin{array}{l}28 \\
27.5\end{array}$ & $\begin{array}{l}\text { na } \\
\text { na }\end{array}$ & \\
\hline $\begin{array}{l}\text { Type I interferon score } \\
\left(\text { Lübbers et } \mathrm{al}^{24}\right)\end{array}$ & & & & $\begin{array}{l}\text { I5/25 IFN Nigh patients converted to arthritis, versus } \\
29 / 90 \text { IFN }{ }^{\text {low }} \text { patients }(P=0.01 \mathrm{I})\end{array}$ \\
\hline $\begin{array}{l}\text { IFN } \\
\text { IFNigh } \\
\text { IFN }\end{array}$ & $\begin{array}{l}\text { na } \\
\text { na } \\
\text { na }\end{array}$ & $\begin{array}{l}\text { na } \\
\text { na } \\
\text { na }\end{array}$ & $\begin{array}{l}60 \\
32.2 \\
38.2\end{array}$ & \\
\hline $\begin{array}{l}\text { STAT3-inducible genes } \\
\left(\text { Pratt et } \mathrm{al}^{15}\right)\end{array}$ & 75 & 85 & na & $\begin{array}{l}\text { Serum IL- } 6 \text { was highest in anti-CCP subjects, which } \\
\text { distinguished this subgroup from non-RA inflammatory } \\
\text { synovitis (corrected } P<0.05 \text { ). Paired IL- } 6 \text { correlated } \\
\text { with STAT3 expression }\end{array}$ \\
\hline $\begin{array}{l}\text { IL-6 } \\
\left(\text { Pratt et al }{ }^{15}\right)\end{array}$ & na & na & na & - \\
\hline
\end{tabular}

Note: *Calculated based on the reported data.

Abbreviations: ACPA, anti-cyclic citrullinated peptide antibody; CCP, cyclic citrullinated peptide; IFN, interferon; IL, interleukin; na, not available; RA, rheumatoid arthritis; Reg, regulatory; Th, T helper.

$39.3 \%$ and specificity of $80.3 \%$, suggesting that presence of a susceptibility marker increases the positive predictive value or odds ratio in favor of the disease. ${ }^{17}$ The meta-analysis suggests HLA $D R B 1 * 0401$ has an odds ratio of 4.41 whereas that for PTPN22 is $1.94 .{ }^{18}$ Increased relative risk of susceptibility improves sensitivity of detection, whereas the strength in causation will improve the specificity. However, given that the prevalence of genes in the community is on the higher side, they are not specific to diagnosis but do improve the positive predictive value of a pathological marker. The association of RA with its susceptibility markers is not very strong. The majority of susceptibility markers have very limited predictability when screened in an asymptomatic population.

\section{Regulatory factors as markers of RA}

RA is an immunological disease. There are characteristic changes expected to earmark the disease process and these changes will be observed to be statistically different from normal. Elevated activated CD8 cells, CD19 B-cells are seen to be present in RA compared to healthy controls. ${ }^{19}$ Reduced levels of regulatory CD4+ CD25 $5^{\text {high }} \mathrm{T}$-cells and T helper 17 CD4+ T-cells have been observed in the case of RA. ${ }^{20,21}$ Similarly, altered T-cell proliferation kinetics, serum 
Table 4 Autoantibody markers of RA with available evidence

\begin{tabular}{|c|c|c|c|c|}
\hline Markers of early RA (reference) & Specificity (\%) & Sensitivity (\%) & Positivity (\%) & Observation/detection \\
\hline RF (IgM-RF) & & & & - \\
\hline \multicolumn{5}{|l|}{$\left(\right.$ Solanki et $\mathrm{a}^{25}$ ) } \\
\hline ( $<2$ years $)$ & na & 57 & na & \\
\hline ( $>2$ years $)$ & na & 81 & na & \\
\hline \multicolumn{5}{|l|}{ (Mouterde et $\mathrm{a}^{26}$ ) } \\
\hline (6 months) & 55 & 70 & 46.3 & \\
\hline (12 months) & 35 & 85 & na & \\
\hline$\left(\right.$ Pawłowska et $\mathrm{al}^{22}$ ) & 56.3 & 70 & na & \\
\hline$\left(\right.$ Liu et $\left.\mathrm{al}^{27}\right)$ & 80.1 & 72.4 & na & \\
\hline (Berglin et $\mathrm{al}^{16}$ ) & 94 & 22 & na & \\
\hline (Johansson et $\mathrm{al}^{17}$ ) & 94.1 & 22 & na & \\
\hline RF (IgG-RF) & & & & - \\
\hline (Berglin et $\left.\mathrm{al}^{16}\right)$ & 94 & 17 & na & \\
\hline (Johansson et $\mathrm{al}^{17}$ ) & 95.8 & 16.9 & na & \\
\hline RF (IgA-RF) & & & & - \\
\hline (Berglin et $\mathrm{al}^{16}$ ) & 94 & 42 & na & \\
\hline (Johansson et $\mathrm{al}^{17}$ ) & 94.9 & 42.4 & na & \\
\hline \multicolumn{5}{|l|}{ Anti-CCP (ACPA) } \\
\hline \multirow[t]{2}{*}{$\left(\right.$ Solanki et $\mathrm{a}^{25}$ ) } & na & 79 (<2 years $)$ & na & $20 \%$ more sensitive than RF, independent \\
\hline & & 84 (>2 years) & & predictor of radiological damage, progression \\
\hline$\left(\right.$ Forslind et $\mathrm{a}^{28}$ ) & na & 64 & na & of disease course over 3 years \\
\hline$\left(\right.$ Kastbom et $\left.\mathrm{al}^{29}\right)$ & na & 93.1 & na & \\
\hline$\left(\right.$ Li et $\left.\mathrm{al}^{30}\right)$ & na & 65 & na & \\
\hline \multicolumn{5}{|l|}{$\left(\right.$ Mouterde et $\mathrm{al}^{26}$ ) } \\
\hline (6 months) & 68 & 65 & 39.4 & \\
\hline (12 months) & 68 & 66 & na & \\
\hline$\left(\right.$ Pawłowska et $\mathrm{al}^{22}$ ) & 66.7 & 80 & na & \\
\hline (Berglin et $\mathrm{al}^{16}$ ) & 98 & 37 & na & \\
\hline (Johansson et $\mathrm{al}^{17}$ ) & 98.6 & 37.1 & na & \\
\hline Anti-CCP2 & & & & - \\
\hline$\left(\right.$ Liu et $\left.\mathrm{al}^{27}\right)$ & 96.3 & 61.8 & na & \\
\hline \multicolumn{5}{|l|}{ (Damjanovska et $\mathrm{al}^{31}$ ) } \\
\hline RA versus early $R A$ & 93.4 & 56.7 & 56.9 & \\
\hline RA versus healthy & 99.0 & 56.9 & 98.9 & \\
\hline$\left(\right.$ Pratt et $\left.\mathrm{al}^{32}\right)$ & 93.0 & 48.0 & na & \\
\hline Anti-CCP3.I & & & & - \\
\hline \multicolumn{5}{|l|}{ (Damjanovska et $\mathrm{al}^{31}$ ) } \\
\hline RA versus early RA & 90.0 & 58.1 & 90.0 & \\
\hline RA versus healthy & 99.0 & 58.1 & 98.9 & \\
\hline
\end{tabular}

\section{Anti-MCV}

(Damjanovska et al ${ }^{31}$ )

RA versus early $R A$

RA versus healthy

82.9

93.9

62.0

62.0

(Liu et $\mathrm{a}^{27}$ )

93.4

78.2

62.0

na

Serotyping for an extended ACPA panel does not add value to CCP2 testing for diagnosing RA in an early undifferentiated arthritis cohort

The diagnostic action of the anti-MCV test in the differential diagnosis of early RA is lower than that of anti-CCP tests

Anti-(ROS modified CII)

(Strollo et a $\left.\right|^{33}$ )

Early RA versus healthy

98

92

Anti-MCV is significantly correlated with antiCCP2 and RF, but not any other factor

\section{Anti-(HOCl-CII)}

(Strollo et $\mathrm{al}^{33}$ )

Early RA versus healthy

$>75$

$>75$

na 
Table 4 (Continued)

\begin{tabular}{|c|c|c|c|c|}
\hline Markers of early RA (reference) & Specificity (\%) & Sensitivity (\%) & Positivity (\%) & Observation/detection \\
\hline $\begin{array}{l}\text { Anti-(Gly-CII) } \\
\left(\text { Strollo et } \mathrm{al}^{33}\right)\end{array}$ & & & & - \\
\hline Early RA versus healthy & 65 & 64 & na & \\
\hline $\begin{array}{l}\text { Anti-(citrullinated HPVP-IgG) } \\
\left(\text { Li et } \mathrm{al}^{30}\right)\end{array}$ & & & & - \\
\hline Early RA versus healthy & 62.4 & 89.4 & 62.3 & \\
\hline $\begin{array}{l}\text { Anti-(citrullinated HPVP-IgM) } \\
\left(\text { Li et } \mathrm{a}^{30}\right)\end{array}$ & & & & - \\
\hline Early RA versus healthy & 66.3 & 86.6 & 66.3 & \\
\hline $\begin{array}{l}\text { Anti-(citrullinated HPVP-IgA) } \\
\left(\text { Li et } \mathrm{al}^{30}\right)\end{array}$ & & & & - \\
\hline Early RA versus healthy & 28.7 & 93.1 & 28.7 & \\
\hline Anti-CB I0 peptide of type II collagen & & & & - \\
\hline$\left(\right.$ Cook et $\left.\mathrm{al}^{34}\right)$ & na & 88 & na & \\
\hline Antinuclear antibody-Hep2 & & & & - \\
\hline (Pawłowska et al ${ }^{22}$ ) & $5 \mathrm{I} .1$ & 4.5 & na & \\
\hline Anti-BiP & & & & - \\
\hline$\left(\right.$ Blass et al ${ }^{35}$ ) & 96 & 63 & na & \\
\hline Anti- $\alpha$-enolase & & & & \\
\hline$\left(\right.$ Saulot et $\left.\mathrm{al}^{36}\right)$ & 97.1 & na & 24.8 & $\begin{array}{l}\text { In healthy individuals and patients with other } \\
\text { rheumatic diseases, BiP-reactive T-cells were } \\
\text { undetectable. BiP overexpression and BiP- } \\
\text { specific autoimmunity may be involved in RA }\end{array}$ \\
\hline
\end{tabular}

Abbreviations: ACPA, anti-cyclic citrullinated peptide antibody; BiP, binding protein; CBI0, cyanogen bromide cleaved peptide 10 of type II collagen; CCP, cyclic citrullinated peptide; CII, type II collagen; Gly, glycation/glycoxidation; Hep2, human epidermoid cancer cells; HOCI, hypochlorous acid; HPVP, human papilloma virus peptide; Ig, immunoglobulin; MCV, mutated citrullinated vimentin; na, not available; RA, rheumatoid arthritis; RF, rheumatoid factor; ROS, reactive oxygen species.

levels of IL-7, and type 1 interferon (IFN) score are seen in rheumatic disease and normal controls. The studies evaluating RA. ${ }^{22-24}$ Though the differences are seen in early RA patients, T-cell kinetics and IFN signature have shown higher sensitivthere are few studies which have evaluated the specificity ity and specificity (Table 3), but there are limited experiences and sensitivity of these differences with reference to other with these tests.

Table 5 Disease features as markers of RA with available evidence

\begin{tabular}{|c|c|c|c|c|}
\hline Markers of early RA (reference) & Specificity (\%) & Sensitivity (\%) & Positivity (\%) & Observation/detection \\
\hline Serum CXCLI 3 & & & & Baseline levels of CXCLI 3 were significantly \\
\hline (Bugatti et $\mathrm{al}^{37}$ ) & & & & higher in RA compared to controls ( $n=19$ ) \\
\hline Low CXCLI3 & na & na & 71 & $(P=0.03)$; linked with measures of synovitis \\
\hline High CXCLI3 & na & na & 59 & \\
\hline $\begin{array}{l}\text { Plasma-ficolin } 3 \\
\left(\text { Roy et a }\left.\right|^{38}\right)\end{array}$ & na & na & na & $\begin{array}{l}\text { Ficolin } 3 \text { is upregulated in the plasma of RA } \\
\text { patients }\end{array}$ \\
\hline $\begin{array}{l}\text { Serum-TTR } \\
\left(\mathrm{Ni} \text { et } \mathrm{al}^{39}\right)\end{array}$ & & & & $\begin{array}{l}\text { TTR in sera of early RA patients were } \\
\text { significantly increased. Four isoforms of TTR }\end{array}$ \\
\hline Overall $(n=149)$ & na & na & 24.2 & varied with different disease stages \\
\hline $\begin{array}{l}\text { ADMA } \\
\left(\text { Di Franco et al }{ }^{40}\right)\end{array}$ & na & na & na & $\begin{array}{l}\text { ADMA levels were significantly higher than } \\
\text { controls at baseline }(P=0.007)\end{array}$ \\
\hline $\begin{array}{l}\text { Apelin } \\
\left(\text { Di Franco et } \mathrm{al}^{40}\right)\end{array}$ & na & na & na & $\begin{array}{l}\text { Serum apelin levels were significantly } \\
\text { decreased versus controls }(P=0.000 \mathrm{I})\end{array}$ \\
\hline $\begin{array}{l}\text { DAS } 44 \text { in ACPA cohort } \\
\left(\text { Goëb et } \mathrm{a}^{23}\right)\end{array}$ & 68 & 61 & na & - \\
\hline
\end{tabular}

Abbreviations: ACPA, anti-cyclic citrullinated peptide antibody; ADMA, asymmetric dimethylarginine; CXCLI3, lymphoid chemokine; DAS, disease activity score; na, not available; RA, rheumatoid arthritis; TTR, transthyretin. 
Table 6 Biomarker combinations with available evidence

\begin{tabular}{|c|c|c|c|c|}
\hline Markers of early RA (reference) & Specificity (\%) & Sensitivity (\%) & Positivity (\%) & Observation/detection \\
\hline RF and anti-CCP & & & & - \\
\hline$\left(\right.$ Niewold et $\left.\mathrm{al}^{43}\right)$ & $99-100$ & & na & \\
\hline$\left(\right.$ Tedesco et $\left.\mathrm{al}^{45}\right)$ & 87 & 100 & na & \\
\hline$\left(\right.$ Panchagnula et $\mathrm{al}^{46}$ ) & 96.1 & 63.4 & na & \\
\hline $\begin{array}{l}\text { Anti-CCP and SE HLA DR allele } \\
\left.\text { (Berglin et } \mathrm{al}^{16}\right)\end{array}$ & & & & - \\
\hline SE HLA + anti-CCP & 99 & 28 & na & \\
\hline
\end{tabular}

Anti-CCP and SNP PTPN22 gene

(Johansson et al ${ }^{17}$ )

Anti-CCP + PTPN22 CT + TT

SE HLA DR + SNP PTPN22 gene

(Johansson et al $^{17}$ )

SE $(B I * 0404$ or $040 I)+P T P N 22 C T+T T$

IgA-RF and SE HLA DR allele

(Berglin et al ${ }^{16}$ )

$$
\text { SE }(B I * 0404 / 040 I)+\text { IgA-RF }
$$

IgM-RF and SE HLA DR allele

(Berglin et $\mathrm{al}^{16}$ )

$$
\text { SE }(B I * 0404 / 040 I)+\text { IgM-RF }
$$

98

IgG-RF and SE HLA DR allele

(Berglin et $\mathrm{al}^{16}$ )

$$
\text { SE }(B I * 0404 / 040 I)+\text { IgG-RF }
$$

SE and SE HLA DR allele

(Berglin et $\mathrm{al}^{16}$ )

SESE

IgG-RF and SESE HLA DR allele

(Berglin et $\mathrm{al}^{16}$ )

SESE $(B I * 0404 / 040 I)+\lg$ G-RF

MCP-I, anti-CCP, RF-IgM, hsCRP, and IL-6

(Rantapää-Dahlqvist et a ${ }^{49}$ )

7 variables - Leiden score

(Visser et al ${ }^{50}$ )

\section{ACR/EULAR criteria}

(Radner et $\mathrm{a}^{42}$ )

Prediction model with $\mathbf{4}$ measures and number of tender and swollen joints

(Kuriya et al ${ }^{54}$ )

IFN ${ }^{\text {high }}$, ACPA, and RF

(Lübbers et $\mathrm{al}^{24}$ )

Anti-MCV and anti-CCP2

(Liu et $\mathrm{a}^{27}$ )

Anti-MCV and anti-CCP2 used as one combined criterion

(Liu et $\mathrm{al}^{27}$ )

97.8

81.2

$35 / 80$

na

na

(score $\geq 8$ )

na

78.5

97.8

58.8

na

IL-7 and ACPA

(Goëb et $\mathrm{a}^{23}$ )

DAS-44 and IL-7 in ACPA

(Goëb et $\mathrm{a}^{23}$ )

na

na

na

na

na

$99 \quad$ II na

$95 \quad 12 \quad$ na
100

22. 1

39.3

na

The combination of the PTPN22 I858T variant and anti-CCP antibodies was found only among pre-RA patients

$-$

$-$

MCP-I levels were found to be significantly raised in patients with anti$\mathrm{CCP}$ and IgM-RF

$-$

$-$

High scores in our cohort predicted progression to RA by 6 months. Baseline scores $\geq 8$ corresponded with higher rates of progression

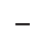


Table 6 (Continued)

\begin{tabular}{|c|c|c|c|c|}
\hline Markers of early RA (reference) & Specificity (\%) & Sensitivity (\%) & Positivity (\%) & Observation/detection \\
\hline \multicolumn{4}{|l|}{ Cytokine profile IL-6/IL-2 I } & IL- 6 and IL-2I were the only cytokines that \\
\hline \multicolumn{4}{|l|}{$\left(\right.$ Gottenberg et $\left.\mathrm{al}^{55}\right)$} & \multirow{2}{*}{$\begin{array}{l}\text { discriminated RA from UA on univariate } \\
\text { analysis. IL-6 level was associated with RA }\end{array}$} \\
\hline IL-6+ and IL-2I+ & 87.7 & 66.4 & 17 & \\
\hline \multicolumn{4}{|l|}{2010 ACR/EULAR criteria } & \multirow[t]{2}{*}{-} \\
\hline (Billiavska et $\mathrm{a}^{56}$ ) & 60 & 80 & 47.5 & \\
\hline \multicolumn{4}{|l|}{9 clinical variables (prediction score) } & \multirow[t]{2}{*}{-} \\
\hline (van der Helm-van Mil et $\mathrm{al}^{57}$ ) & 94 & 100 & 47 & \\
\hline \multirow{3}{*}{\multicolumn{4}{|c|}{$\begin{array}{l}\text { Anti-CCP+ and/or IgM-RF+, MRI-proven } \\
\text { symmetric synovitis, MRI-proven bone } \\
\text { edema and/or bone erosion }\end{array}$}} & \multirow{4}{*}{$\begin{array}{l}\text { Plain MRI is effective in identifying bone } \\
\text { lesions in the wrist and finger joints in } \\
\text { early RA, but not synovitis }\end{array}$} \\
\hline & & & & \\
\hline & & & & \\
\hline$\left(\right.$ Tamai et $\left.\mathrm{al}^{58}\right)$ & 75.9 & 68 & 71.3 & \\
\hline \multirow{2}{*}{\multicolumn{4}{|c|}{$\begin{array}{l}\text { A prediction model - clinical hand arthritis, } \\
\text { RF+, morning stiffness, bone edema on }\end{array}$}} & \multirow[t]{4}{*}{-} \\
\hline & & & & \\
\hline \multicolumn{4}{|l|}{ MRI in the MTP and wrist joints } & \\
\hline (Duer-Jensen et $\mathrm{a}^{59}$ ) & 82 & 81 & 60.2 & \\
\hline
\end{tabular}

\section{Autoantibody markers}

Currently, autoantibodies to RF and CCP are well established markers of RA. Numerous studies have evaluated the utility of anti-RF, ${ }^{16,17,22,25-27}$ anti-CCP, ${ }^{16,17,22,26-32}$ and their variants. Antibodies to modified type II collagens, ${ }^{33}$ degradation products of type II collagen, ${ }^{34}$ vimetin, ${ }^{27,31}$ stress-related proteins, ${ }^{35}$ and other peptides ${ }^{30,36}$ have also been explored recently. The specificity and sensitivity of a few autoantibodies are presented in Table 4. A single autoantibody has a sensitivity ranging from $30 \%-70 \%$ and a few of them have a better specificity. Use of a single antibody as a marker increases sensitivity, while a combination of them increases specificity. In spite of this, $10 \%-30 \%$ of RA patients in early stages of the disease may not have any defined autoantibody, making it difficult to predict impending RA based on autoantibodies.

\section{Degradation products and secreted products}

The degradation products and secreted products, like $\mathrm{C}-\mathrm{X}-\mathrm{C}$ motif chemokine 13 (CXCL13), at sites of inflammation can serve as a marker of pathology. ${ }^{37}$ Similarly, elevated ficolin 3 , which is a humoral molecule of the innate immune system that recognizes carbohydrate molecules on pathogens, are differentially elevated in patients of RA. ${ }^{38}$ Similarly, transthyretin, a thyroxine binding protein, is elevated in early RA. ${ }^{39}$ Another metabolic product, asymmetric dimethyl-arginine, has been shown to be elevated in comparison to healthy con- trols, but is not a specific marker of arthritis. Reduced serum apelin has also been observed in a few patients. ${ }^{40}$

\section{Combinations of biomarkers}

The heterogeneity of the disease, explained above, precludes the argument that a single biomarker could be specific as well as sensitive. Development of combined predictors or biomarkers should fulfill the obligation of being specific as well as sensitive. We analyze here the possibility of utilization of combined biomarkers and the available literature to support the concept (Table 6).

\section{Combination of autoantibodies}

The American College of Rheumatology (ACR) criteria, which incorporate both clinical and laboratory parameters in diagnosis of RA, are more sensitive and specific than individual features put together. The recently developed ACR criteria have been estimated to possess an overall sensitivity of $82 \%$ and specificity of $61 \%$ when applied to the intended target population. ${ }^{41,42}$ The rate of identification of RA increases when RF and anti-CCP are used separately with the clinical features. This is one of the reasons for the increased sensitivity of newer ACR/European League Against Rheumatism (EULAR) criteria. Studies have reported 5\%-13\% of patients to be positive for anti-CCP in RF-negative RA population. Thus, if anti-CCP is used separately, there is a possibility of diagnosing an additional $10 \%$ of RA-suspected patients. ${ }^{43}$ In isolation, RF and anti-CCP have lower specificity. A recent 
meta-analysis suggested a pooled sensitivity and specificity of 67\% (confidence interval [CI] 65-68) and 95\% (CI 95-96), respectively, for anti-CCP antibody, and 69\% (CI 68-70) and 85\% (CI 84-86) for immunoglobulin (Ig)M-RF. ${ }^{44}$ However, when both RF and anti-CCP are combined, specificity increases to $99 \%-100 \%{ }^{43}$ In a novel study using combined multiplex cytofluorimetric assay, combination of anti-CCP with RF appeared to be more sensitive (100\%) and specific (87\%) than the nephelometric assay for RF detection. ${ }^{45}$ The greatest challenge is when patients do not have all the clinical characteristics typical of RA. The specificity and sensitivity of anti-CCP in such a case were $96.1 \%$ and $63.4 \%$, respectively. ${ }^{46}$ The antibodies like anti-CCP, RF-IgA, RF-IgM, and RF-IgG appear in a significant proportion of RA patients, much before the development of clinical disease. ${ }^{7}$ Even the specificity for the prediction of the onset of RA in a pre-arthritis sample increased when two antibodies - anti-CCP and an RF isotype - were combined while the sensitivity was reduced.

Thus, the combination of biomarkers may enhance the specificity at the cost of sensitivity, but this can be compensated by using matrices where an adequately weighted scoring system is used both individually and in combination. The choice of combination and use of multiple autoantibodies are expected to enhance both sensitivity and specificity. Antibodies against carbamylated fetal calf serum (anti-Ca-FCS) and carbamylated-fibrinogen (anti-Ca-Fib) were present to the extent of $27 \%$ and $38 \%$, respectively, in serum samples of blood donors prior to the diagnosis of RA. Both anti-Ca-FCS and anti-Ca-Fib could be detected many years before the onset of RA. ${ }^{47}$ Anti-carbamylated protein antibodies as well as ACPA are, on average, detected earlier than IgM-RF. ${ }^{48}$

\section{Combination of biomarkers from different pathological processes}

\section{Genetic susceptibility and Auto-antibody positivity}

Anti-CCP and genetic markers individually do not have high specificity and relative risk prediction. Berglin et al have attempted to analyze the predictability of development of RA using a combination of an autoantibody (anti-CCP) and a shared epitope allele: HLA DRB1*0401 or B1*0404 (SE HLA DR). ${ }^{16}$ They have demonstrated increased predictability as well as improved specificity in the prediction of RA. In the same way, a combination of a single nucleotide polymorphism in the PTPN22 gene encoding the lymphoid protein tyrosine phosphatase and the presence of a detectable level of anti-CCP have shown improved predictability for the development of RA and also the increased specificity of prediction. ${ }^{17}$ The prediction is superior to SE HLA DR alone or in combination. The presence of PTPN22 $1858 \mathrm{~T}$ variant $(\mathrm{CT}+\mathrm{TT})$ and anti-CCP had a specificity of $100 \%$ with almost 20-times increased strength of predictability. These studies highlight the fact that the presence of susceptibility markers improves the positive predictive value of an add-on test.

\section{Addition of activation markers}

Chemotactic activation marker monocyte chemotactic protein-1 (MCP1) was increased with the onset of clinical disease and elevation of high-sensitivity $\mathrm{C}$-reactive protein (hsCRP) and IL-6. ${ }^{49}$ In a study identifying the predictors of RA in undifferentiated arthritis, markers like serum IL-7 and ACPA, and their combination with disease activity score (DAS) of 44, increased the specificity of prediction. ${ }^{23}$ The changes, like reduced $\mathrm{T}$ helper 17 cells, IFN signature, and reduced regulatory $\mathrm{CD} 4+\mathrm{CD} 25^{\text {high }} \mathrm{T}$-cells in autoantibody positive patients, exhibit an increased specificity. ${ }^{20,21,24}$

\section{Clinical features and lab parameters}

Leiden score, which includes both clinical and lab parameters, helps in predicting the probability of undifferentiated arthritis progressing to RA. The score consists of seven variables: symptom duration at first visit; morning stiffness for $>1$ hour; arthritis in more than three joints; bilateral compression pain in the metatarsophalangeal joints; RF positivity; anti-CCP positivity; and the presence of erosion in hands/ feet. ${ }^{50}$ The newer ACR/EULAR criteria could diagnose RA earlier than previous ACR criteria, but still lacks the desired sensitivity and specificity. In a meta-analysis of these criteria, pooled sensitivity and specificity for RA (defined by different reference standards) were 0.82 (95\% CI 0.79-0.84) and 0.61 (95\% CI 0.59-0.64), respectively. ${ }^{42}$ The increased sensitivity of newer criteria is at the cost of specificity, evident on comparison with the previous criteria. In a follow-up study of patients categorized as having undifferentiated inflammatory arthritis, 24\% developed RA. Anti-CCP and Leiden score were able to categorize only $6 \%$ and $1 \%$ into the group that predicted development of RA, respectively. ${ }^{51}$ Observations such as this one point to the fact that inclusion of nonspecific and nondiscriminatory markers, either biological markers or clinical features, can improve sensitivity but with a reduction in specificity.

\section{Differential specification should be picked from the pathways}

RA is a heterogeneous and dynamic disease. A single or a pooled biomarker may not be beneficial for the entire RA 
population, especially in the early course of the disease. The question of utmost importance is to identify the best markers and to choose them for further studies. Hence, it is preferable to select factors or molecules that are specific to RA pathogenesis and present in a significant proportion of RA population. For instance, utilization of a cartilage degradation marker as an additional marker for diagnosis and differentiation of RA did not remarkably contribute to diagnosis, since they are elevated in any form of inflammatory synovitis. The high diagnostic specificity of anti-CCP antibodies for RA was observed in a prospective population-based study of very early arthritis. Raised serum cartilage oligomeric matrix protein levels were common in all diagnosis groups in this series, indicating cartilage degradation is not unique to RA. The specificity was better and significant for antiCCP, relative to cartilage oligomeric matrix protein. ${ }^{52}$ The inflammatory markers, like hsCRP, represent activation of inflammation and suffer from nonspecificity; hence, they will not be suitable as diagnostic markers. This fact is evidenced in a Finnish study where 19,072 C-reactive protein levels were demonstrated to be of no predictive value in patients expected to develop RA. ${ }^{53}$

\section{Conclusion}

A dynamic disease like RA is unlikely to have a single, unique biomarker to diagnose or predict the development of disease. A selected factor or molecule should be specific to RA, sensitive to the progression of disease, and cover a wide range of RA patients in order to qualify as a suitable biomarker. Only a combination of factors appears to fulfill all of these requirements. Some of the promising biomarker combinations include: change in immunological parameters like cell kinetics with the appearance of autoantibodies; specific cytokine changes; and other markers of disease susceptibility. Multicenter studies exploring these factors should help improve their quality as biomarkers of early RA.

\section{Acknowledgment}

The author is grateful to Research Assist (http://www. research-assist.com) for assistance with manuscript editing and preparation.

\section{Disclosure}

The author reports no conflicts of interest in this work.

\section{References}

1. Finckle A, Liang MH, van Herckenrode CM, de Pablo P. Long term impact of early treatment on radiological progression in rheumatoid arthritis: A meta-analysis. Arthritis Rheum. 2006;55:864-872.
2. van Aken J, van Dongen $\mathrm{H}$, le Cessie S, Allaart CF, Breedveld FC, Huizinga TW. Comparison of long term outcome of patients with rheumatoid arthritis presenting with undifferentiated arthritis or with rheumatoid arthritis: an observational cohort study. Ann Rheum Dis. 2006;65:20-25.

3. Hazes JM, Luime JJ. The epidemiology of early inflammatory arthritis. Nat Rev Rheumatol. 2011;7:381-390.

4. Machado P, Castrejon I, Katchamart W, et al. Multinational evidence-based recommendations on how to investigate and follow-up undifferentiated peripheral inflammatory arthritis: integrating systematic literature research and expert opinion of a broad international panel of rheumatologists in the 3E Initiative. Ann Rheum Dis. 2011;70(1):15-24.

5. Olivieri I, Sarzi-Puttini P, Bugatti S, Atzeni F, d'Angelo S, Caporali R. Early treatment in early undifferentiated arthritis. Autoimmune Rev. 2012;11(8):589-592.

6. Chandrashekara S. Pharmacokinetic consideration of synthetic DMARDs in rheumatoid arthritis. Expert Opin Drug Metab Toxicol. 2013;9(8):969-981.

7. Rantapää-Dahlqvist S, de Jong BA, Berglin E, et al. Antibodies against cyclic citrullinated peptide and $\operatorname{IgA}$ rheumatoid factor predict the development of rheumatoid arthritis. Arthritis Rheum. 2003;48(10):2741-2749.

8. Weyand CM, Goronzy JJ. Association of MHC and rheumatoid arthritis. HLA polymorphisms in phenotypic variants of rheumatoid arthritis. Arthritis Res. 2000;2(3):212-216.

9. Kang YM, Zhang X, Wagner UG, et al. CD8 T cells are required for the formation of ectopic germinal centers in rheumatoid synovitis. J Exp Med. 2002;195:1325-1336.

10. Gorman JD, Lum RF, Chen JJ, Suarez-Almazor ME, Thomson G, Criswell LA. Impact of shared epitope genotype and ethnicity on erosive disease: a meta-analysis of 3,240 rheumatoid arthritis patients. Arthritis Rheum. 2004;50(2):400-412.

11. Schotte H, Schlüter B, Drynda S, et al. Interleukin 10 promoter microsatellite polymorphisms are associated with response to long term treatment with etanercept in patients with rheumatoid arthritis. Ann Rheumatic Dis. 2005;64(4):575-581.

12. Sen M. Wnt signalling in rheumatoid arthritis. Rheumatology (Oxford). 2005;44(6):708-713.

13. Marinou I, Healy J, Mewar D, et al. Association of interleukin-6 and interleukin-10 genotypes with radiographic damage in rheumatoid arthritis is dependent on autoantibody status. Arthritis Rheum. 2007;56(8):2549-2556.

14. Kallberg H, Padyukov L, Plenge RM, et al. Gene-gene and gene-environment interactions involving HLA-DRB1, PTPN22 and smoking in two subsets of rheumatoid arthritis. Am J Hum Genet. 2007;80: 867-875.

15. Pratt AG, Swan DC, Richardson S, et al. A CD4 T cell gene signature for early rheumatoid arthritis implicates interleukin 6-mediated STAT3 signalling, particularly in anti-citrullinated peptide antibody-negative disease. Ann Rheum Dis. 2012;71:1374-1381.

16. Berglin E, Padyukov L, Sundin U, et al. A combination of autoantibodies to cyclic citrullinated peptide (CCP) and HLA-DRB1 locus antigens is strongly associated with future onset of rheumatoid arthritis. Arthritis Res Ther. 2004;6(4):R303-R308.

17. Johansson M, Arlestig L, Hallmans G, Rantapää-Dahlqvist S. PTPN22 polymorphism and anti-cyclic citrullinated peptide antibodies in combination strongly predicts future onset of rheumatoid arthritis and has a specificity of $100 \%$ for the disease. Arthritis Res Ther. 2006;8(1):R19.

18. Scott IC, Seegobin SD, Steer S, et al. Predicting the risk of rheumatoid arthritis and its age of onset through modeling genetic risk variants with smoking. PLoS Genet. 2013;9(9):e1003808.

19. van Baarsen LG, de Hair MJ, Ramwadhdoebe TH, et al. The cellular composition of lymph nodes in the earliest phase of inflammatory arthritis. Ann Rheum Dis. 2013;72:1420-1424.

20. Lawson CA, Brown AK, Bejarano V, et al. Early rheumatoid arthritis is associated with a deficit in the $\mathrm{CD} 4^{+} \mathrm{CD} 25^{\text {high }}$ regulatory $\mathrm{T}$ cell population in peripheral blood. Rheumatology (Oxford). 2006;45:1210-1217.

21. Arroyo-Villa I, Bautista-Caro MB, Balsa A, et al. Frequency of th 17 CD4+ T cells in early rheumatoid arthritis: a marker of anti-CCP seropositivity. PLoS One. 2012;7(8):e42189. 
22. Pawłowska J, Smoleńska Ż, Zdrojewski Z, Witkowski JM, Bryl E. Changes in proliferation kinetics of $T$ cells: A new predictive cellular biomarkers for early rheumatoid arthritis? J Clin Immunol. 2012;32: 991-999.

23. Goëb V, Aegerter P, Parmar R, et al. Progression to rheumatoid arthritis in early inflammatory arthritis is associated with low IL-7 serum levels. Ann Rheum Dis. 2013;72:1032-1036.

24. Lübbers J, Brink M, van de Stadt LA, et al. The type I IFN signature as a biomarker of preclinical rheumatoid arthritis. Ann Rheum Dis. 2013;72:776-780.

25. Solanki K, Spellerberg M, Chapman P, Moller P, O’Donnell J. Anti-cyclic citrullinated antibodies: complementary to IgM rheumatoid factor in the early diagnosis of rheumatoid arthritis. $N \mathrm{Z} \mathrm{Med} \mathrm{J}$. 2004;117(1203):U1097.

26. Mouterde G, Lukas C, Logeart I, et al. Predictors of radiographic progression in the ESPOIR cohort: the season of first symptoms may influence the short-term outcome in early arthritis. Ann Rheum Dis. 2011;70(7):1251-1256.

27. Liu X, Jia R, Zhao J, Li Z. The role of anti-mutated citrullinated vimentin antibodies in the diagnosis of early rheumatoid arthritis. J Rheumatol. 2009;36(6):1136-1142.

28. Forslind K, Ahlmén M, Eberhardt K, Hafström I, Svensson B; BARFOT Study Group. Prediction of radiological outcome in early rheumatoid arthritis in clinical practice: role of antibodies to citrullinated peptides (anti-CCP). Ann Rheum Dis. 2004;63:1090-1095.

29. Kastbom A, Strandberg G, Lindroos A, Skogh T. Anti-CCP antibody test predicts the disease course during 3 years in early rheumatoid arthritis (the Swedish TIRA project). Ann Rheum Dis. 2004;63:1085-1089.

30. Li GY, Sun XL, Li Y, Jia RL, Jia Y, Li ZG. [Significance of anti-citrullinated human papilloma virus-47 E2(345-362) peptide antibodies in diagnosis of early-stage rheumatoid arthritis]. Beijing Da Хие Хие Вао. 2013;45(3):458-463. Chinese.

31. Damjanovska L, Thabet MM, Levarth EW, et al. Diagnostic value of anti-MCV antibodies in differentiating early inflammatory arthritis. Ann Rheum Dis. 2010;69:730-732.

32. Pratt AG, Charles PJ, Chowdhury M, Wilson G, Venables PJ, Isaacs JD. Serotyping for an extended anti-citrullinated peptide autoantibody panel does not add value to CCP2 testing for diagnosing RA in an early undifferentiated arthritis cohort. Ann Rheum Dis. 2011;70: 2056-2058.

33. Strollo R, Ponchel F, Malmström V, et al. Autoantibodies to posttranslationally modified type II collagen as potential biomarkers for rheumatoid arthritis. Arthritis Rheum. 2013;65(7): 1702-1712

34. Cook A, Rowley M, Mackay M, inventors; Montech Medical Developments Pty Ltd, assignee. Method for detecting rheumatoid arthritis employing the CB10 peptide of mammalian type II collagen as an antigen. United States patent US 6706490 B1. March 16, 2004.

35. Blass S, Union A, Raymackers $\mathrm{J}$, et al. The stress protein BiP is overexpressed and is a major $\mathrm{B}$ and $\mathrm{T}$ cell target in rheumatoid arthritis. Arthritis Rheum. 2001;44(4):761-771.

36. Saulot V, Vittecoq O, Charlionet R, et al. Presence of autoantibodies to the glycolytic enzyme alpha-enolase in sera from patients with early rheumatoid arthritis. Arthritis Rheum. 2002;46(5):1196-1201.

37. Bugatti S, Manzo A, Benaglio F, et al. Serum levels of CXCL13 are associated with ultrasonographic synovitis and predict power Doppler persistence in early rheumatoid arthritis treated with nonbiological disease-modifying anti-rheumatic drugs. Arthritis Res Ther. 2012;14:R34.

38. Roy S, Biswas S, Saroha A, Sahu D, Das HR. Enhanced expression and fucosylation of ficolin3 in plasma of RA patients. Clin Biochem. 2013;46(1-2):160-163.

39. Ni M, Wei W, Feng Q, et al. Transthyretin as a potential serological marker for the diagnosis of patients with early rheumatoid arthritis. Clin Experimental Rheumatol. 2013;31:394-399.
40. Di Franco M, Spinelli FR, Metere A, et al. Serum levels of asymmetric dimethylarginine and apelin as potential markers of vascular endothelial dysfunction in early rheumatoid arthritis. Mediators Inflamm. 2012;2012:347268.

41. Zeidler H. Systemic literature review of the performance of the 2010 ACR/EULAR classification criteria for rheumatoid arthritis: good news of debatable significance. Ann Rheum Dis. 2013;72(8):e21.

42. Radner H, Neogi T, Smolen JS, Aletaha D. Performance of the 2010 ACR/EULAR classification criteria for rheumatoid arthritis: a systematic literature review. Ann Rheum Dis. 2014;73(1):114-123.

43. Niewold TB, Harrison MJ, Paget SA. Anti-CCP antibody testing as a diagnostic and prognostic tool in rheumatoid arthritis. QJM. 2007:100(4):193-201.

44. Nishimura K, Sugiyama D, Kogata Y, et al. Meta-analysis: diagnostic accuracy of anti-cyclic citrullinated peptide antibody and rheumatoid factor for rheumatoid arthritis. Ann Intern Med. 2007;146(11): 797-808.

45. Tedesco A, D'Agostino D, Soriente I, Amato P, Piccoli R, Sabatini P. A new strategy for the early diagnosis of rheumatoid arthritis: a combined approach. Autoimmun Rev. 2009;8(3):233-237.

46. Panchagnula R, Rajiv SR, Prakash J, Chandrashekara S, Suresh KP. Role of anti-cyclic citrullinated peptide in the diagnosis of early rheumatoid factor-negative suspected rheumatoid arthritis: is it worthwhile to order the test? J Clin Rheumatol. 2006;12(4):172-175.

47. Shi J, van de Stadt LA, Levarht EW, et al. Anti-carbamylated protein antibodies are present in arthralgia patients and predict the development of rheumatoid arthritis. Arthritis Rheum. 2013;65(4):911-915.

48. Shi J, van de Stadt LA, Levarht EW, et al. Anti-carbamylated protein (anti-CarP) antibodies precede the onset of rheumatoid arthritis. Ann Rheum Dis. 2014;73(4):780-783.

49. Rantapää-Dahlqvist S, Boman K, Tarkowski A, Hallmans G. Up regulation of monocyte chemoattractant protein-1 expression in anticitrulline antibody and immunoglobulin $\mathrm{M}$ rheumatoid factor positive subjects precedes onset of inflammatory response and development of overt rheumatoid arthritis. Ann Rheum Dis. 2007;66(1):121-123.

50. Visser H, le Cessie S, Vos K, Breedveld FC, Hazes JM. How to diagnose rheumatoid arthritis early: a prediction model for persistent (erosive) arthritis. Arthritis Rheum. 2002;46(2):357-365.

51. Krabben A, Abhishek A, Britsemmer K, et al. Risk of rheumatoid arthritis development in patients with unclassified arthritis according to the 2010 ACR/EULAR criteria for rheumatoid arthritis. Rheumatology (Oxford). 2013;52(7):1265-1270.

52. Söderlin MK, Kastbom A, Kautiainen H, Leirisalo-Repo M, Strandberg G, Skogh T. Antibodies against cyclic citrullinated peptide (CCP) and levels of cartilage oligomeric matrix protein (COMP) in very early arthritis: relation to diagnosis and disease activity. Scand $J$ Rheumatol. 2004;33(3):185-188.

53. Aho K, Palosuo T, Knekt P, Alha P, Aromaa A, Heliövaara M. Serum C-reactive protein does not predict rheumatoid arthritis. J Rheumatol. 2000;27(5):1136-1138.

54. Kuriya B, Cheng CK, Chen HM, Bykerk VP. Validation of a prediction rule for development of rheumatoid arthritis in patients with early undifferentiated arthritis. Ann Rheum Dis. 2009;68:1482-1485.

55. Gottenberg JE, Dayer JM, Lukas C, et al. Serum IL-6 and IL-21 are associated with markers of B cell activation and structural progression in early rheumatoid arthritis: results from the ESPOIR cohort. Ann Rheum Dis. 2012;71:1243-1248.

56. Billiavska I, Stamm TA, Martinez-Avila J, et al. Application of the 2010 ACR/EULAR classification criteria in patients with very early inflammatory arthritis: analysis of sensitivity, specificity and predictive values in the SAVE study cohort. Ann Rheum Dis. 2013;72:1335-1341.

57. van der Helm-van Mil AH, le Cessie S, van Dongen H, Breedveld FC, Toes RE, Huizinga TW. A prediction rule for disease outcome in patients with recent-onset undifferentiated arthritis: how to guide individual treatment decisions. Arthritis Rheum. 2007;56(2):433-440. 
58. Tamai M, Kawakami A, Uetani M, et al. Magnetic resonance imaging (MRI) detection of synovitis and bone lesions of the wrists and finger joints in early-stage rheumatoid arthritis: comparison of accuracy of plain MRI-based findings and gadolinium-diethylenetriamine pentaacetic acid-enhanced MRI-based findings. Mod Rheumatol. 2012;22(5):654-658.
59. Duer-Jensen A, Hørslev-Petersen K, Hetland ML, et al. Bone edema on magnetic resonance imaging is an independent predictor of rheumatoid arthritis development in patients with early undifferentiated arthritis. Arthritis Rheum. 2011;63(8):2192-2202.

\section{Publish your work in this journal}

Current Biomarker Findings is an international, peer-reviewed, open access journal publishing original research, reports, reviews and commentaries on all areas of biomarker research. The manuscript management system is completely online and includes a very quick and fair

\section{Dovepress}

peer-review system. Visit http://www.dovepress.com/testimonials.php to read real quotes from published authors. 